\title{
Optical coherence tomography imaging of collagenous tissue microstructure
}

\author{
Kristi A. Hansen, Jennifer K. Barton, Jeffrey A. Weiss \\ Biomedical Engineering Program, University of Arizona, Tucson, AZ 85721
}

\begin{abstract}
The engineering of new biomaterials requires an in-depth understanding of the structure and function of the native tissues. Optical coherence tomography (OCT) is a non-destructive technique that allows the visualization of the microstructure of biological tissues. The aim of this study was to determine if OCT could be used to identify geometric properties of tendons such as crimp pattern. Freshly harvested tendon tissue was imaged with OCT during the application of incremental axial strain. Loads were simultaneously recorded during imaging. Results revealed that birefringent banding perpendicular to the collagen fiber axis could be visualized and measured. This crimp banding disappeared at a very low strain. Birefringent banding parallel to the collagen fiber axis was also seen and it was observed that the number of parallel bands increased as strain increased. Stress-strain data was calculated and found to lie within the expected range. These results indicate that OCT may prove to be a useful tool for the non-destructive analysis of tissue microstructure.
\end{abstract}

Keywords: OCT, collagen, crimp, birefringence

\section{INTRODUCTION}

To engineer new biomaterials or induce the generation of new tissue, the properties and processes of the native tissue must first be understood. The relationship between tissue microstructure and continuum level function has long been a topic of interest in biomechanics and tissue engineering. Mechanical properties of biological tissues depend on structural organization and features, from the microscopic to macroscopic level. Due to the difficulties in accurately visualizing the microstructure, studies of structure-function relations in biological soft tissues are often difficult or impossible. Although histological methods can provide some microstructural information, the techniques require destruction of the tissue and often alter the relationship between tissue components.

Tendons and ligaments are small flexible pieces of fibrous connective tissue. Tendons join muscle to bone and ligaments join bone to bone, transmitting force and guiding movement. These soft tissues are commonly injured and heal by scarring, which results in altered interior tissue organization and inferior mechanical properties when compared to control tissues. Surgical reconstruction or replacement is often required. Understanding the behavior of tendons and ligaments is important in order to prevent injuries, optimize reconstruction, and improve replacements. Both tendons and ligaments are composed mainly of type I collagen. Collagen molecules form into microfibrils that aggregate to form subfibrils. A number of subfibrils form fibrils, which associate into fibers or fascicles ${ }^{1}$. Fascicles finally assemble to form tendons and ligaments. Fascicles can be viewed under polarized light to reveal a longitudinal waveform or crimp pattern. In rat tail tendon fascicles, the crimp pattern on the surface was observed to disappear under low stress ${ }^{2,3}$. It is generally accepted that the stress-strain curve for collagenous tissue is not linear, but consists of three regions, the first of which is called the toe region, in which collagen fibers gradually straighten ${ }^{4}$. It is believed that the transition from the toe region to the second region, which is linear, is the point at which the crimp pattern has completely straightened or disappeared. The third region is again nonlinear, due to the onset of internal failure, and ends at complete failure. It is of interest to note that the physiological function of tendon is mostly confined to the toe region ${ }^{5}$.

Recent attention has been given to non-destructive techniques used to quantify the microstructure of soft tissues. Sacks, Smith, and Hiester ${ }^{6}$ developed a small angle light scattering device that can measure fiber orientation in soft tissues of less than $500 \mu \mathrm{m}$ thickness with an angular resolution of approximately 1 degree and a spatial resolution of $+/-254 \mu \mathrm{m}$. Magnetic resonance diffusion tensor techniques have been used to measure fiber orientation in canine myocardium ${ }^{7}$ and the lamellar structure of the intervertebral $\mathrm{disc}^{8}$. Others have developed non-invasive techniques to investigate the composition 
of the different layers of skin ${ }^{9}$. Optical Coherence Tomography (OCT) provides a relatively inexpensive, non-destructive alternative for the analysis of soft tissue microstructure, with resolution that is potentially superior to the aforementioned techniques in three dimensions (on the order of $15 \mu \mathrm{m})^{10}$. OCT is an optical analogue to ultrasound, utilizing reflected nearinfrared light instead of sound. The intensity of reflected low coherence light is measured, using a Michelson interferometer to determine its spatial origin (Figure 1). It is a recently developed technology that has been used to image many biological tissues in vitro or in vivo, including skin ${ }^{11}$, retina ${ }^{12}$, oviduct ${ }^{13}$, aorta ${ }^{14}$ and tendon ${ }^{15}$.

The birefringent nature of collagen enables the visualization of crimp pattern under polarized light in the form of light and dark bands perpendicular to the collagen fiber axis. Birefringence previously seen in OCT images, however, appears to be indicative of tissue state $^{16}$. Collagenous tissues observed under polarization sensitive OCT (PS-OCT) lose birefringence when they are damaged ${ }^{17}$ or disorganized ${ }^{18}$. Boer ${ }^{15}$ used PS-OCT to image fresh bovine tendon, revealing banding parallel to the fiber axis. Tendons are highly organized even in their relaxed state, resulting in the appearance of birefringence effects in OCT images, even using OCT that is not polarization sensitive. As tendon is stretched, the fibers straighten and become more perfectly aligned with the tendon axis. This has been reported to correlate with changes in the crimp pattern of the collagen.

Nondestructive and potentially noninvasive measurement of the ultrastructure of collagenous soft tissues would provide a unique method for the evaluation of normal and healing tissues and the efficacy of treatment regimens. The technique could also be used to determine microstructural parameters for constitutive models of the tissue material behavior ${ }^{19,20}$. The objectives of this work were to assess the feasibility of quantifying the crimp pattern of tendons under different states of strain. Our hypotheses were that OCT imaging would allow visualization of tendon crimp pattern because of its birefringent nature, and that the birefringence would be extinguished as successively increasing increments in tensile strain were applied to the tendons.

\section{METHODS}

\subsection{Optical coherence tomography}

The OCT system used to image tendons in this study was recently built by the investigators and is similar to one described by Izatt $^{10}$ (Figure 1). The system has axial resolution of $15 \mu \mathrm{m}$ in air and lateral resolution of approximately $15 \mu \mathrm{m}$. Acquisition time varies depending on the number of pixels in the image. Based on an image 512 x 100 pixels, acquisition time is approximately 7 seconds. The system uses a superluminescent source with a center wavelength of $1295 \mathrm{~nm}$. Crosssectional scans are taken into the tissue one to two millimeters and along the tissue the desired length, dependent on the movement of a motorized stage (Figure 2).

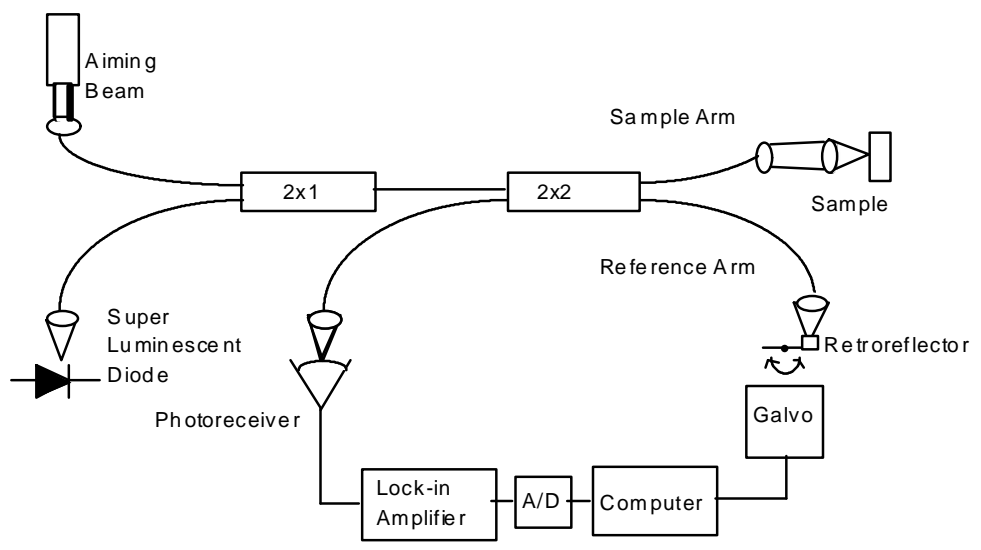

Figure 1: OCT system. Backscattered light from the sample and translating retroreflector interferes when the pathlength is within the coherence length of the source. 


\subsection{Tissue preparation}

Tendons from the forelimb of a mature sheep were obtained immediately following slaughter. Rat tail tendons from four Sprague Dawley rats were obtained immediately following sacrifice. All tendons were kept continuously moist with $0.9 \%$ buffered saline during harvest and preparation, and images were acquired within 48 hours of sacrifice. Sheep tendons were excised from the surrounding tissue and trimmed to a length of approximately $35 \mathrm{~mm}$. The tendons were gripped in stainless steel clamps and mounted in a custom-built stretching apparatus (Figure 2). The skin was removed from the rat tails and pieces of tendon were carefully dissected away from the proximal end of the tail. Single fascicle specimens were teased out of the tendon section. All rat tail fascicles were secured with cyanoacrylate between two small pieces of rubber to increase the gripping area and avoid tissue damage at the clamped area. Nylon thread markers were attached transverse to the collagen fiber direction of the tissue with cyanoacrylate, on specimens longer than $20 \mathrm{~mm}$ (Figure 2, inset). Each end was then placed in a metal clamp, allowing for a small amount of slack to keep from prematurely stretching the tissue. The stretching apparatus was then attached to the motorized stage and the saline bath was filled to just cover the tissue.

\subsection{Mechanical testing}

The sheep tendons were used in the initial development and validation of the experimental protocol, while the rat tendons were used to correlate crimp with the stress-strain behavior of the tissue. The width and thickness of rat tail specimens were measured using digital calipers and the cross-sectional area was determined using the assumption of elliptical cross-sectional geometry. Thickness measurements were later verified using values determined from the OCT images. The nylon markers formed a gage length for strain measurements using images acquired by the OCT system (Figure 2). In rat tail fascicle specimens that were shorter than $20 \mathrm{~mm}$, clamp edges were imaged to form the gage length. One clamp was attached to a translation stage and the other was fixed to a stationary plate. A micrometer adjusted the position of the translation stage to apply strain to the clamped tissue. The stretching apparatus was attached to a computer-controlled motorized stage to allow movement along the collagen fiber axis for OCT image acquisition. The zero-load length of the tissue was established by consecutively applying and removing a small tare load via adjustment of the micrometer. The clamp-to-clamp distance was then measured with calipers. An image of the tissue with zero-load length was acquired in the vertical plane with the OCT system. Using the measured zero-load length, the rat tail fascicle was consecutively stretched to lengths corresponding to 1 , 2, 3, 4 and 5\% clamp-to-clamp strain or 30-50 $\mu \mathrm{m}$ increments. The exact tissue-level strain for the rat tail fascicles was later determined from the change in distance between the nylon thread markers in the OCT images. A $100 \mathrm{lb}$. load cell (Transducer Techniques, Temecula, CA), integrated into the stretching apparatus, was connected to an amplifying circuit and then interfaced with a data acquisition system. The load was continuously monitored and recorded during the application of each strain increment, and an OCT image was acquired for each strain increment when the change in load dropped below a value of $0.03 \mathrm{~N} / \mathrm{sec}$. Engineering stress, $\sigma$, was calculated at each strain increment using the initial cross-sectional area $A_{0}$ and the measured load $F$ as $\sigma=F / A_{0}$.

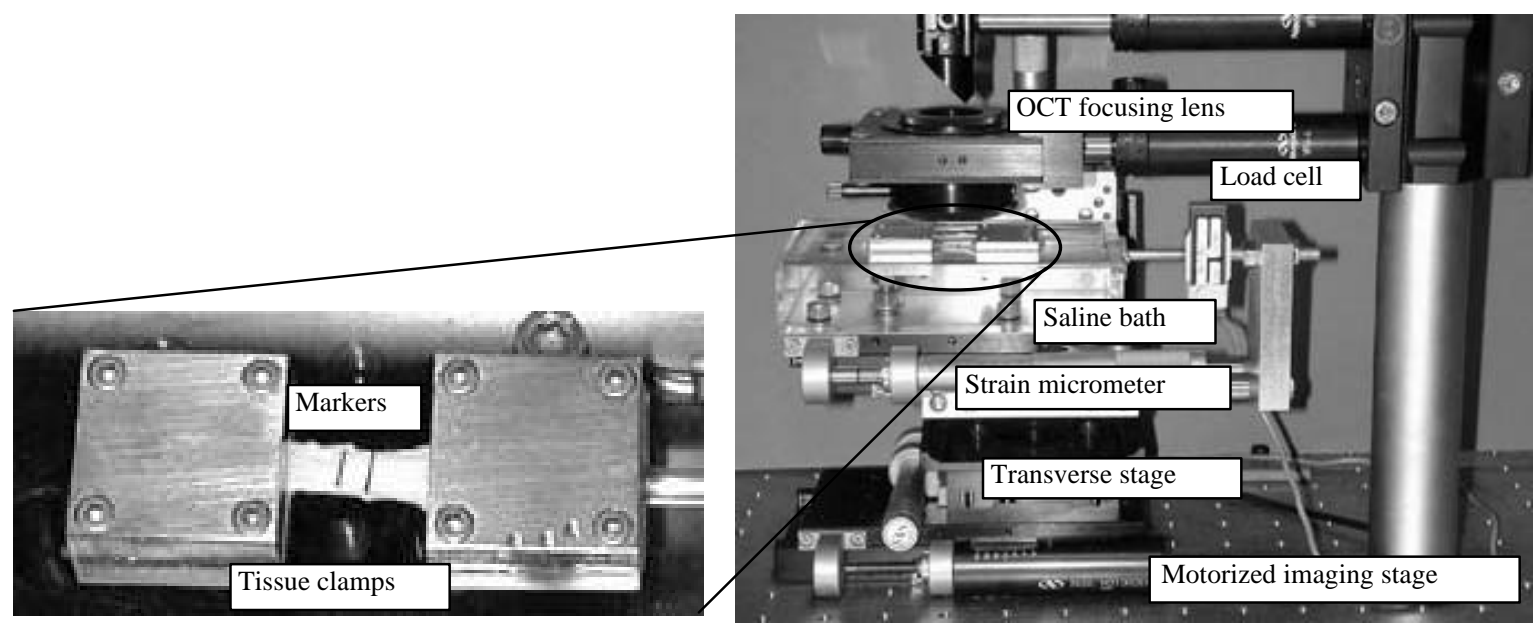

Figure 2: Stretching apparatus with enlarged top view of tissue clamps holding sheep tendon. 


\subsection{Data analysis}

Strain and crimp period were measured using image analysis software (Scion Inc., Frederick, MD). Marker-to-marker distances were measured to determine the strain on the tissue portion being imaged. Engineering strain was calculated as $\varepsilon=$ $\Delta L / L_{0}$, where $L_{0}$ is the marker-to-marker distance on the unstrained tissue and $\Delta L$ is the difference between the strained marker-to-marker distance and $L_{0}$. Crimp period, identified by one dark and light band pair, was measured over the imaged length and ranges were reported.

\section{RESULTS}

\subsection{Crimp pattern and birefringence changes}

Preliminary tests on sheep tendons show how the ultrastructure of the tendon differed under different strain conditions. In Figure 3, both vertical and horizontal banding is seen in the interior of the tendon. The vertical banding is believed to represent the collagen birefringence due to crimp, while the vertical banding is likely a part of the structural organization of the tendon. As strain was applied to the tissue, the vertical banding gradually disappeared from the images (Figure 4). The dark region in the top center of the tissue is due to dehydration of the tissue. The black nylon markers placed on the top surface of the tissue absorb the light and cause the thick dark vertical stripes seen in the images.

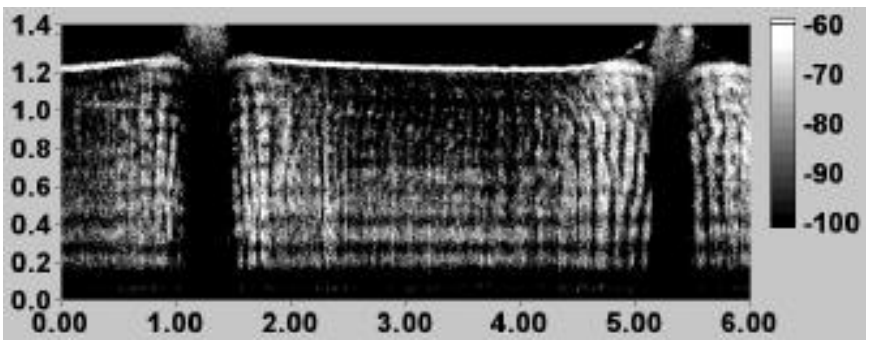

Figure 3: Sheep tendon before strain application. Vertical banding indicative of collagen crimp pattern is apparent.

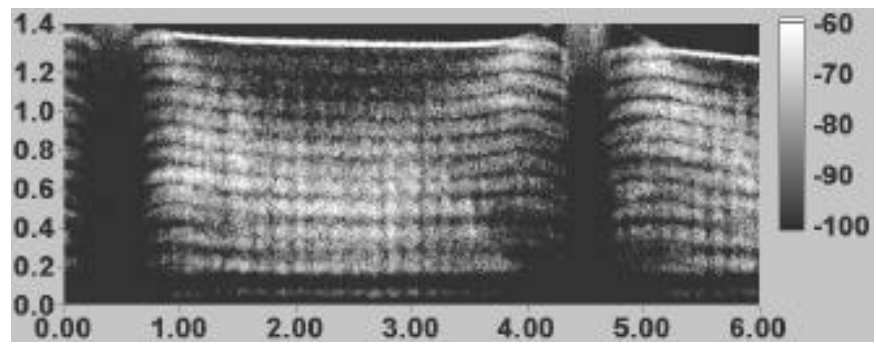

Figure 4: Sheep tendon after application of $2.4 \%$ strain. Vertical banding disappeared and horizontal banding became more apparent.

Figure 5 represents typical images resulting from the rat tail fascicles tests, as a sequence. Measurable crimp period ranged from $100 \mu \mathrm{m}$ to $200 \mu \mathrm{m}$ in Figures 5A-C. Crimp period in Figure 5D ranged from $100 \mu \mathrm{m}$ to $160 \mu \mathrm{m}$. Crimp banding disappeared in Figure 5E. There is little change between images A, B, and C, the first two increments of strain. All three images show distinct vertical and horizontal banding. The third increment shows decreased visibility of crimp banding and an additional bright horizontal band appears. Larger crimp periods seen in the previous images have disappeared and only small crimp periods remain in Figure 5D. In the final image the crimp banding can no longer be distinguished and the new horizontal band in the previous image has increased in thickness.

\subsection{Stress-strain data}

Stress strain data for single fascicle tests is plotted in Figure 6. Values are within the expected range. 
Proc. SPIE BIOS 2000 - Biomedical Optics Symposium, Vol 3914, January 2000.

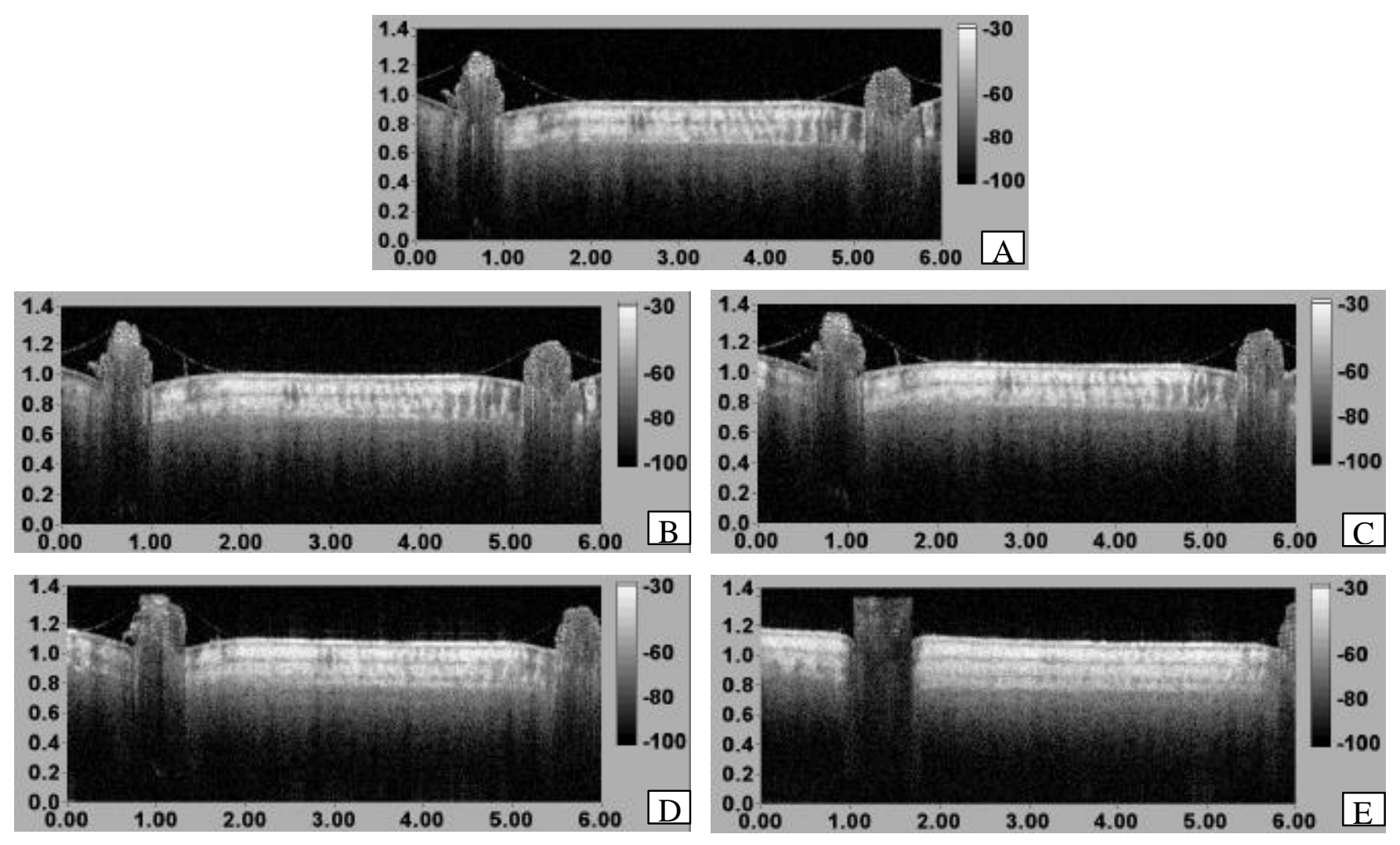

Figure 5A-E: Change in birefringent banding with increasing strain. Clamp-to-clamp strain: A) 0.0\%, B) $0.4 \%$, C) $0.8 \%$, D) $1.3 \%$, E) $1.7 \%$.

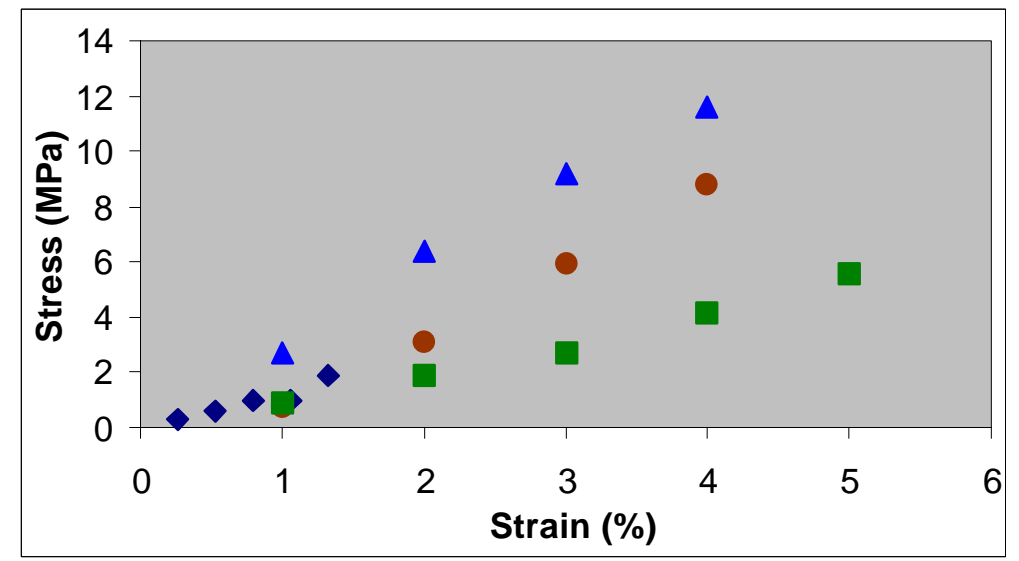

Figure 6: Stress-strain data for tests of rat tail tendon fascicles.

\section{DISCUSSION}

This study demonstrated that OCT imaging can visualize microstructural details of collagenous tissues. In particular, the crimp pattern of tendons was readily visible in the OCT images, and changes in the crimp pattern were seen as the tissue was stretched. Preliminary OCT images of sheep tendon showed the horizontal banding like that seen by other researchers ${ }^{15}$ 
using PS-OCT to look at bovine tendon, and in addition revealed the vertical birefringent banding indicative of crimp pattern. Knowing that crimp pattern is believed to disappear at low strain, we desired to find if OCT could image this process. Also, understanding that birefringent horizontal banding indicates tissue state or level of organization, we wanted to see if the horizontal banding changed as the tendon was strained. Prior to these tests, we introduced a polarization-rotating element into the sample arm of the system. Changes in the banding appearance were observed as the element was rotated, assuring us that the banding observed was indeed a polarization effect.

Figure 5A-E shows the progressive change in birefringent banding of rat tail tendon fascicle. Relaxed crimp period measurements are in the same range as the $120 \mu \mathrm{m}$ measurement made by Diamant ${ }^{3}$. We attribute the change in the appearance of the banding to the removal of the crimp. We attribute the increased brightness and number of horizontal bands to the increased level of organization that strain induces in the tissue by pulling the fibrils into more parallel alignment with the tensile axis.

A comparison of clamp-to-clamp and marker-to-marker strain was not presented herein. Under very low strain measurements, the two measurements correlate well. At higher strains, the measurements differed in some of the tests. It was observed that the markers in these tests were adhered to the very thin fascia that covered the fascicle. The stretching of this fascia often did not appear to correlate with the stretching of the tendon tissue beneath it, accounting for the difference in measurements. Clamp-to-clamp strain values were used in these cases. In future work the thin fascia will be removed prior to testing.

Stress-strain data for the rat tail tendon fascicles was within the range reported in the literature ${ }^{21}$ of 0 to $20 \mathrm{MPa}$ for strains of 0 to $5 \%$. This suggests that our stress and strain measurements were accurate and provide a good reference between collagen crimp period, applied strain and resulting stress in the tissue. This information, combined with images taken at smaller strain increments, can provide accurate detail on the state of the tissue as it passes through the toe region and transitions into the linear region. Three-dimensional images would be even more useful in determining tissue state, measuring crimp period and measuring cross-sectional areas. Work is presently in progress to develop a real-time OCT imaging system that would then be capable of taking continuous images as tissue is mechanically loaded.

\section{ACKNOWLEDGEMENTS}

This work was supported in part by a grant from the National Science Foundation (KAH, JKB) and a Whitaker Foundation Biomedical Research Grant (JAW).

\section{REFERENCES}

1. J. Kastelic, A. Galeski, and E. Baer, "The multicomposite structure of tendon," Connective Tissue Research, 6:11-23, 1978.

2. B. J. Rigby, N. Hirai, J.D. Spikes, H. Eyring, "The mechanical properties of rat tail tendon," Journal of General Physiology, 43:265289, 1959.

3. J. Diamant, A. Keller, E. Baer, M. Litt, and R. G. C. Arridge, "Collagen; ultrastructure and its relation to mechanical properties as a function of ageing," Proceedings of the Royal Society of London. Series B: Biological Sciences, 180:293-315, 1972.

4. Y. Lanir, E. L. Salant, and A. Foux, "Physico-chemical and microstructural changes in collagen fiber bundles following stretch invitro," Biorheology, 25:591-603, 1988.

5. A. Viidik, C. C. Danielsen, and H. Oxlund, "On fundamental and phenomenological models, structure and mechanical properties of collagen, elastin and glycosaminoglycan complexes," Biorheology, 19:437-451, 1982.

6. M. S. Sacks, D. B. Smith, and E. E. Hiester, "A small angle light scattering device for planar connective tissue microstructural analysis,” Annals of Biomedical Engineering, 25:678-689, 1997.

7. E. W. Hsu, A. L. Muzikant, S. A. Matulevicius, R. C. Penland, and C. S. Henriquez, "Magnetic resonance myocardial fiber-orientation mapping with direct histological correlation," American Journal of Physiology, 274(5 Pt 2):H1627-34, 1998.

8. E. W. Hsu and L. A. Setton, "Diffusion tensor microscopy of the intervertebral disc anulus fibrosus," Magnetic Resonance in Medicine, 41:992-999, 1999.

9. P. J. Caspers, G. W. Lucassen, R. Wolthuis, H. A. Bruining, and G. J. Puppels, "In vitro and in vivo Raman spectroscopy of human skin,” Biospectroscopy, 4(5 Suppl):S31-39, 1998.

10. J. A. Izatt, M. D. Kulkarni, S. Yazdanfar, J. K. Barton, and A. J. Welch, "In vivo bi-directional color Doppler flow imaging of picoliter blood volumes using optical coherence tomography," Optics Letters, 22:1439-1441, 1997.

11. J. M. Schmitt, M. Yadlowsky, and R. F. Bonner, "Subsurface imaging of living skin with optical coherence microscopy," Dermatology, 191:93-98, 1995. 
Proc. SPIE BIOS 2000 - Biomedical Optics Symposium, Vol 3914, January 2000.

12. M. R. Hee, J. A. Izatt, E. A. Swanson, D. Huang, C. P. Lin, J. S. Schuman, C. A. Puliafito, and J. G. Fujimoto, "Optical coherence tomography of the human retina," Archives of Opthalmology, 113:325-332, 1995.

13. J. M. Herrmann, M. E. Brezinski, B. E. Bouma, S. A. Boppart, C. Pitris, J. F. Southern, and J. G. Fujimoto, "Two- and threedimensional high-resolution imaging of the human oviduct with optical coherence tomography," Fertility and Sterility, 70:155-158, 1998.

14. M. E. Brezinski, G. J. Tearney, B. E. Brett, S. A. Boppart, M. R. Hee, E. A. Swanson, J. F. Southern, J. G. Fujimoto, "Imaging of coronary artery microstructure with optical coherence tomography," American Journal of Cardiology, 77:92-93, 1996.

15. J. F. de Boer, T. E. Milner, M. J. C. van Gemert, and J. S. Nelson, "Two-dimensional birefringence imaging in biological tissue by polarization-sensitive optical coherence tomography," Optics Letters, 22:934-936, 1997.

16. M. J. Everett, K. Schoenenberger, B. W. Colston, Jr., and L. B. Da Silva, "Birefringence characterization of biological tissue by optical coherence tomography," Optics Letters, 23:228-230, 1998.

17. S. M. Srinivas, J. F. de Boer, B. H. Park, K. Keikhanzadeh, H. L. Huang, Z. Chen, J. S. Nelson, "Determination of burn depth by polarization sensitive optical coherence tomography," in Coherence Domain Optical Methods in Biomedical Science and Clinical Applications III, Valery V. Tuchin, Joseph A. Izatt, Editors, Proceedings of SPIE Vol. 3598, 70-78, 1999.

18. X. Li, J. Herrmann, R. Ghanta, C. Pitris, W. Drexler, C. Jesser, D. Stamper, D. Golden, S. Martin, J. G. Fujimoto, M. Brezinski, “ OCT imaging of osteoarthritic cartilage: structure, polarization sensitivity, and clinical feasibility," in Coherence Domain Optical Methods in Biomedical Science and Clinical Applications III, Valery V. Tuchin, Joseph A. Izatt, Editors, Proceedings of SPIE Vol. 3598, 152-157, 1999.

19. M. K. Kwan and S. L. Woo, "A structural model to describe the nonlinear stress-strain behavior for parallel-fibered collagenous tissues," ASME Journal of Biomechanical Engineering, 111:361-363, 1989.

20. C. Hurschler, B. Loitz-Ramage, R. Vanderby, Jr., "A structurally based stress-stretch relationship for tendon and ligament," ASME Journal of Biomechanical Engineering, 119:392-399, 1997.

21. J. P. Price, G. O. Njus, T. A. Conway, "Ultrastructural properties of rat tail tendon," Biomedical Engineering Conference. Proceedings of the 1996 Fifteenth Southern, 456-459, 1996. 